\title{
Design and Implementation of Video Monitoring System for Deer Shed Based on Embedded Technology
}

\author{
Min Zhang1,a,Yuhua Wu1,b,Ji Li1,c,Shijun Li1,d \\ 1Jilin Agricultural University, Changchun 130118, Jilin, China \\ a1165339875@qq.com, bawuyuhua@163.com,c735485328@qq.com,dlsj0883@sina.com
}

\begin{abstract}
This paper designs the deer shed video monitoring system based on embedded. By using the embedded technology and video coding technology, applying the real-time camera to collect deer shed on-site information, and then via the video coding technology, analysing and displaying the received video data, real-time monitoring deer's living surroundings, to prevent the emergence of deer Panic, frightening, fighting and other accidents, timely and effectively avoid the economic losses caused by deer's abortion, death, squeezing and so on.
\end{abstract}

Keywords: Embedded, video monitoring system, deer breeding

\section{Introduction}

China has a long history of deer breeding, the earliest can be traced back to the Shang and Zhou dynasties, is one of the world's earliest deer raising countries[1]. As the deer breeding automation, scientific degree is not high, although the Western countries deer farming much later than our country, its velvet exports have been occupying a high international market share [2], far more than China's velvet international market share. Therefore, through the use of Internet of Things technology on the deer breeding automation, scientific improvement has a great role, can improve the level of deer breeding.

With the constantly development of the Internet of things technology, livestock breeding is more and more modern. Video monitoring technology is becoming more and more extensively used in livestock breeding, the remote video monitoring of deep sea cage culture by using 3G and VSaaS technology is proposed in literature [3]; In the literature [4], through the use of dual cameras on the pigpen for video data collection, monitoring its behavior; In the literature [5], through the use of video monitor sauries surface and underwater state; In the literature[6] designed based on Android smart terminal breeding management assistant. In the literature[7], through the use of embedded technology, video surveillance technology, image processing technology for automatic counting of eggs.

In this paper, by using embedded technology and video processing technology of real-time monitoring of deer shed herd life information, to avoid deer shed in panic, frightened, fighting and so on, So as to avoid the economic losses caused by crushing and death, and improve the economic output of deer breeding.

\section{Overall System Framework}

Deer shed video monitor overall system block diagram is shown in figure 1, the appropriate position of the deer shed is arranged with a camera, which is connected with the USB port of the embedded terminal, wherein the embedded terminal adopts a ARM9 development board. The deer's real-time video information transmission to the embedded terminal, and then through the gateway connected to an external database, transmitting the deer living information to the PC side, to facilitate the real-time management of the deer living conditions. When the deer in the herd to frighten panic, fights and other accidents, managers can make corresponding processing in time, avoiding the resulting crush and death, thus reduce the economic losses in the deer breeding process. 


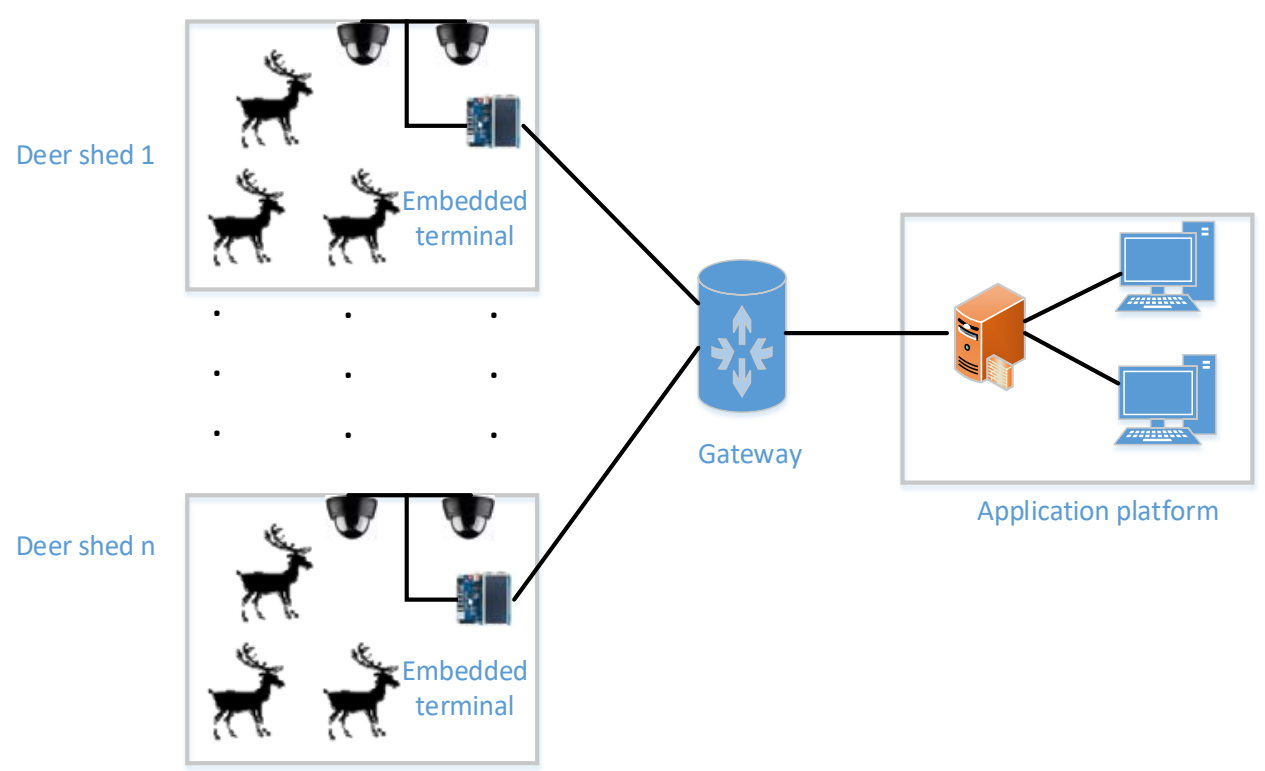

Fig. 1. The overall system framework

\section{Hardware design framework}

The overall hardware design of the system is shown in Figure 2, Mainly include SDRAM, Nor Flash, Nand Flash, USB1, DM9000, Camera, USB2, Serial port, Touch screen, Power supply module. USB1 and the camera to connect to facilitate communication between the camera and S3C2440 processor; SDRAM, Nor Flash, Nand Flash can be seen as embedded terminal storage module; DM9000 is mainly responsible for connecting with the external network, transmitting the deer information through the network to the application platform to facilitate the remote management to view; The main function of USB2 and serial port is to facilitate the developers to debug the development board, through the PC and the connection to the U-Boot system, Linux system, V4L2 driver, YASS2 system transplanted to the development board, so that it can work properly; Touch screen is mainly responsible for deer shed show in video images.

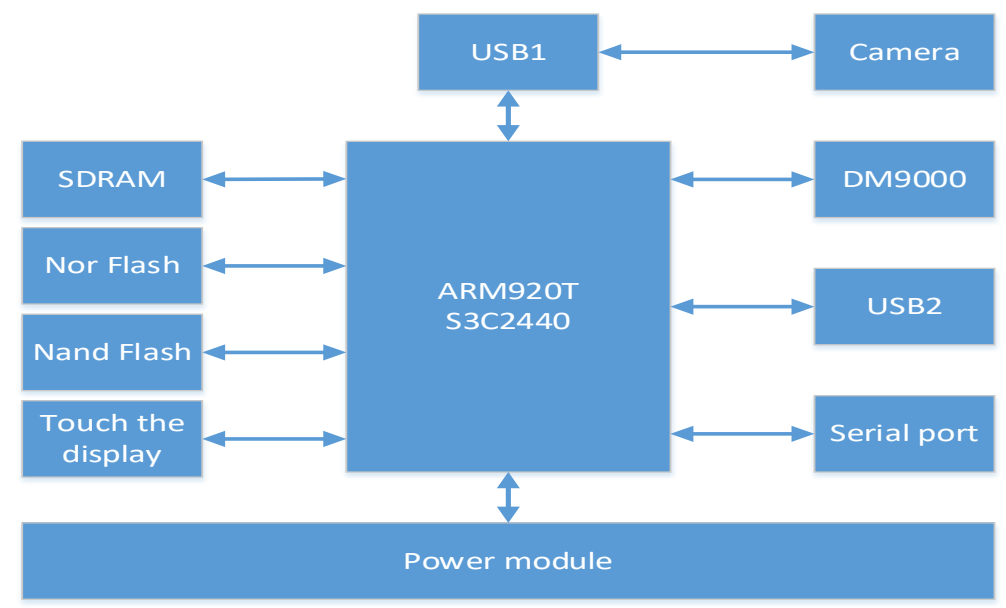

Fig. 2.Hardware overall design block diagram 


\section{Video capture software design}

V4L2 is a Linux device driver framework which is mainly used to deal with the video information. It provides a set of API interface for Linux video access. The application only need to call the corresponding API function can operate different video equipment, the use of V4L2 greatly facilitate the development and maintenance of video[8]. The structure of V4L2 in Linux is shown in Figure 3. The application only needs to call the corresponding device number, the character device driver in the Linux system will call the V4L2 driver layer, and then call the hardware device through the V4L2 layer to achieve the entire video processing.

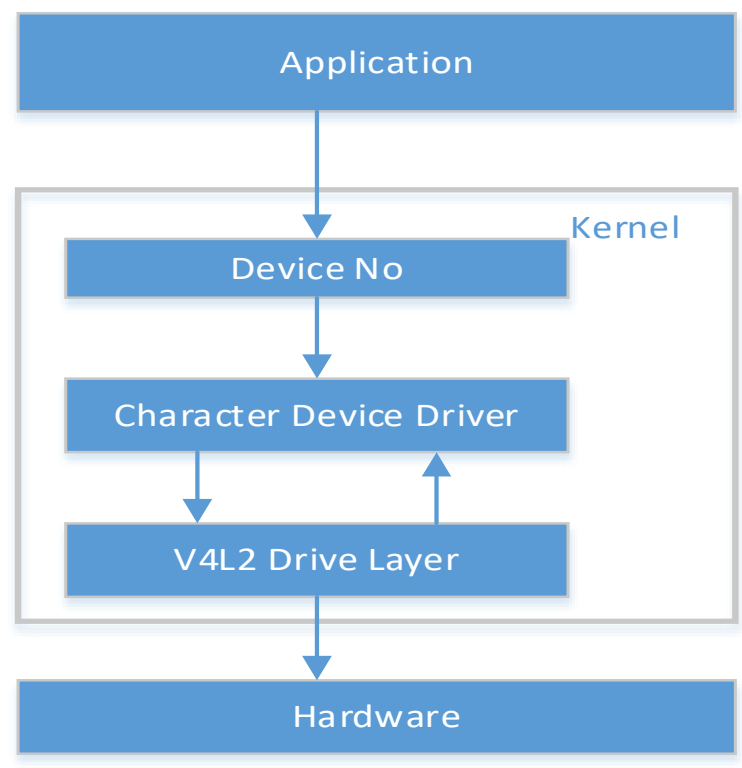

Fig. 3.V4L2 drive structure diagram

The main steps of V4L2 video capture are as follows:

1) Open the device and get the appropriate device file descriptor. There are two types of applications that open video devices: blocking mode and non blocking mode.Compared with non blocking mode, the full transmission of data can be ensured by using blocking mode.

2) Get the equipment capability, Observe whether the device has the function of video input, audio input and output.

3) Set the corresponding video format, Such as PAL, NTSC, height, width and other information.

4)Apply the corresponding frame buffer to the driver,and through the mmap () function will be applied to the frame buffer mapped to the user space, so no need to go to the data replication, can directly capture the collected frame data.

5)Start video capture,The driver will put the buffer data into the queue, and the application will read data from the queue, so the cycle of video capture.

6)When you encounter VIDIOC_STREAMOFF stop command, then stop the collection of video information, and use the close () function to turn off the video device.

V4L2 video capture flow chart shown in Figure 4. 


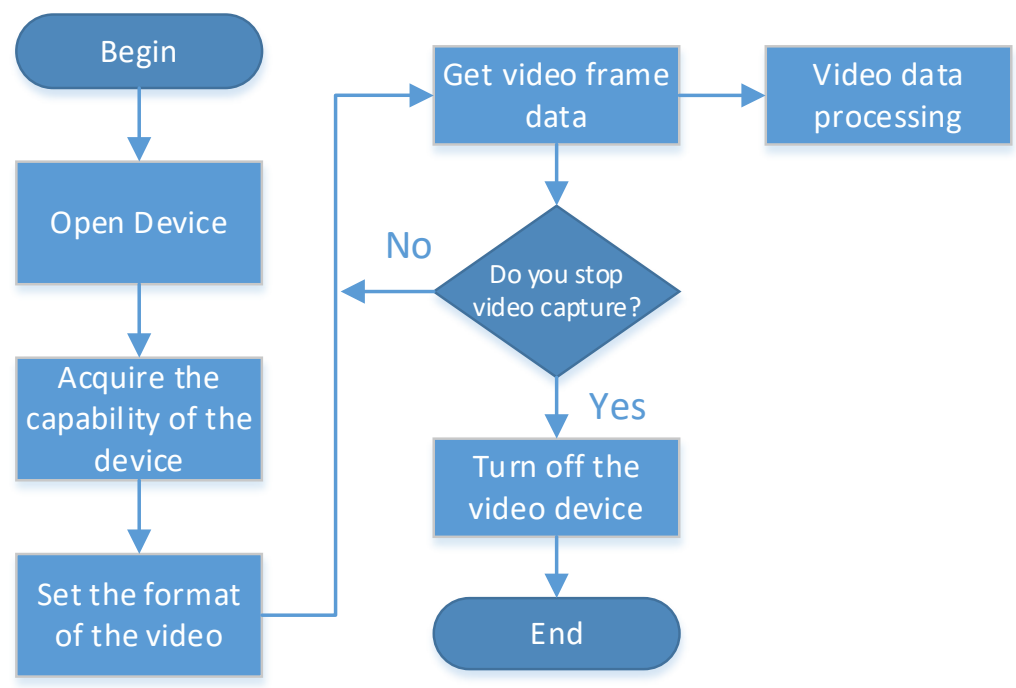

Fig. 4.Video Acquisition Flowchart Based on V4L2

\section{Conclusions}

This paper designs the deer shed video monitoring system based on embedded. Video monitoring through the deer shed, can avoid the deer due to panic, fear, fights and other reasons caused the death, crushing and other accidents, so as to reduce the economic losses in the process of deer breeding.

\section{Acknowledgments}

The authors wish to express their gratitude to the projects:Design and Implementation of Video monitoring System for Deer Shed Based on Embedded from Education Department of Jilin Province, Jilin Province Economic Structural Adjustment Leading Fund Special Project (No. 2014Y108) and Changchun City Science and Technology Plan Project (No. 14nk029), Key Tackling Item of Jilin Province Science \& Technology Department (No. 20140204045NY), for their generous support of this work. At the same time, the other authors also thank the corresponding author, Shijun LI, for his assistance in submitting this paper.

\section{References}

[1]Jiang Yifei, Li Feng, Zhang Shidong, Cheng Wenjuan, Li Xiaopeng, Yu Ming, Liu Haomin. Present situation and Prospect of sika deer industry in China[J]. Shandong Agricultural Sciences, 2012,(09):109-111+114.

[2]Zhang Zhenxing. Prospect and Development Strategy of Deer Industry in China[J]. Journal of Economic Animal Science, 2003,7(2):1-4.

[3]Hu Yu, Guo Genxi, Huang Xiaohua, Tao Qiyou. Remote video surveillance system for deepwater cage culture based on 3G + VSaaS technology[J]. Southern Fisheries Science, 2013,(02):63-69. 
[4]Yang Wei, Yu Shouhua. Application of video surveillance technology in large - scale breeding of pig[J]. Modern agricultural equipment, 2014,(04):37-41.

[5]Tang Lu, Guo Qiang, Guo jia, Zhang Kanyu. Design and implementation of remote monitoring system of the network of saury artificial breeding material[J]. industrial control computer, 2015,(05):51-52+54.

[6]Liu Boping, Hu Min, Yang Guoqiang, Tang Hui, Fu Kang.Based on Android smart terminal breeding management assistant[J]. Computing Technology and Automation, 2016,(04):112-116.

[7]Wang Ling, Wang Chaofeng, Zhang Kui. Design of network video surveillance system for laying hens farms based on ARM[J]. Journal of Agricultural Mechanization, 2012,(02):186-191.

[8]Wei Dongshan. Embedded Linux application development complete manual[M]. Beijing:People Post Press,2008. 